\title{
Variability Survey with the HST
}

Werner W. Weiss, Rainer Kuschnig, Konstanze Zwintz

Institute for Astronomy, University Vienna, A-1180

Türkenschanzstrasse 17. Austria

\begin{abstract}
We report on the analysis of HST archival data obtained with the Fine Guidance Sensors concerning a magnitude limited survey of microvariability among guide stars.
\end{abstract}

\section{Introduction}

The Fine Guidance Sensors (FGS) of the HST provide high precision photometric data of a brightness limited sample of stars which allows the investigation of microvariability primarily on time scales of minutes up to few hours. The scheduling policy for the HST usually does not allow the continuous observation of the same target over many orbits. Hence, FGS datasets which would allow detection of periods considerably longer than several days are rare, as is indicated by Fig. 1.

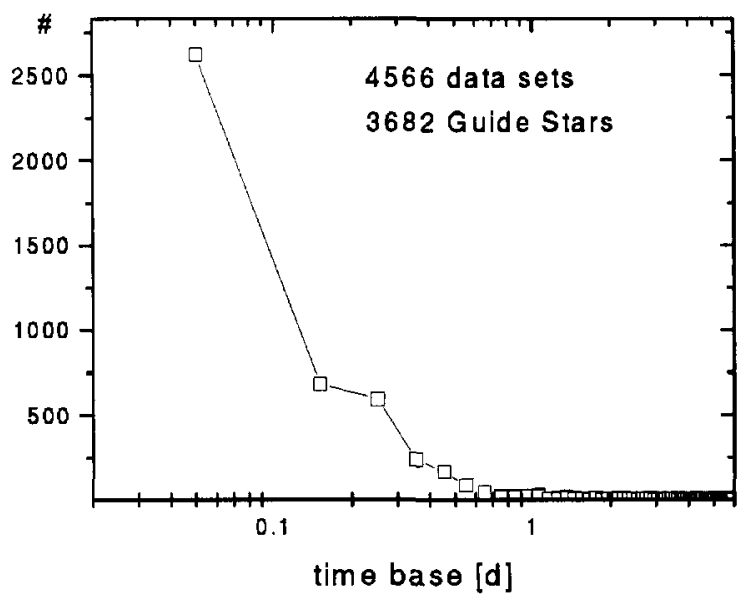

Figure 1. Histogram of datasets as function of time base.

On the other hand, an integration time of $25 \mathrm{~ms}$ allows the study of variability phenomena of extremely short periods - a unique feature of this data body compared to the variability studies based on microlensing surveys also reported during this conference. The latter typically produce only few photometric data points per night. 

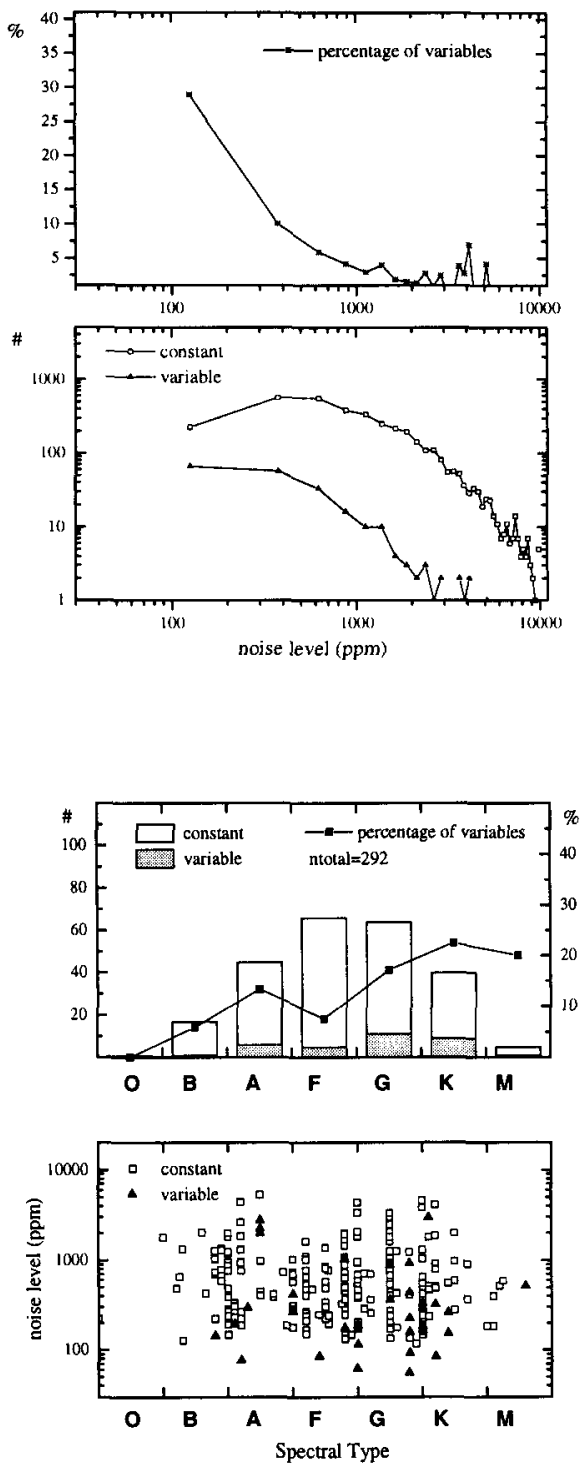

Figure 2. The fraction of variable guide stars found as a function of the noise level (upper two panels). The distribution of variable guide stars as a function of spectral type (lower two panels). 
The instrumental properties were described in Kuschnig et al. (1997) along with the data handling method and the variability criterion used by us for the survey. The influence of stray light, the orbit of the HST, and in particular the South Atlantic Anomaly are discussed in Zwintz et al. (1999a). We also tried to transform the FGS (single color) photometry to Johnson $V$ values and determined deadtime corrections, sensitivity differences of the three Fine Guidance Sensors, and color sensitivity (Weiss et al. 1999). A summary was also presented as a poster during this conference (Zwintz et al. 2000).

\section{The Survey}

For more than 5400 HST-FGS datasets obtained from about 3600 guide stars covering the observing seasons from 1992 August to 1995 November, we have performed time series analyses, based on a Discrete Fourier Transform algorithm developed by Deeming (1975) for unequally spaced data. More than 200 stars of this sample were found to be variable to a high confidence level. Because the HST guide stars are limited in brightness from $V=9$ to 15 , spectral types are known only for a small subset of this sample. As a consequence, we have initiated a spectrum classification observing program for guide stars found to be photometrically interesting.

Fig. 2 illustrates that the percentage of guide stars which are variable increases considerably if the noise level decreases in the Fourier domain, computed as an average of the amplitude spectrum from $\nu=0$ up to the Nyquist frequency. Fig. 2 also indicates a tendency that cooler stars in the present sample from the HST-FGS archive are more likely to be variable than hotter stars. The statistics are based on the first $10 \%$ of the available data.

The first set of variable guide stars which is submitted for publication (Zwintz et al. 1999b) contains a detailed description of our procedures and a frequency solution for the light curves. Based on a SIMBAD database search, on dedicated Strömgren photometry and on spectroscopy we try to provide a classification for the type of variability.

Acknowledgments. Research was supported by a grant S7303-AST of the Austrian Fonds zur Förderung der wissenschaftlichen Forschung and grant 8014 of the Jubiläumsfonds der Österreichischen Nationalbank.

\section{References}

Deeming, T. J. 1975, A\&AS, 36, 137

Kuschnig, R., Weiss, W. W., Gruber, R., Bely, P. Y., \& Jenkner, H. 1997, A\&A, 328,544

Weiss, W. W., Zwintz, K., Kuschnig, R., \& Witeschnik, A. 1999, Comm. Asteroseismology, No. 129

Zwintz, K., Kuschnig, R., Weiss, W. W., Gray, R. O., \& Jenkner, H. 1999a, A\&A, 343, 899

Zwintz, K., Weiss, W. W., \& Kuschnig, R. 1999b, submitted

Zwintz, K., Kuschnig, R., Weiss, W. W., \& Witeschnik, A. 2000, in these proceedings, p. 82 